\title{
Clinical features and management experience in patients with metastatic spinal bladder cancer: a single-institution 10-year retrospective study
}

\author{
Xi Zhou ${ }^{1 \#}$, Shuzhong Liu ${ }^{1 \#}$, Siyuan Yao ${ }^{1}$, Zhen Huo ${ }^{2}$, Yipeng Wang', Yong Liu' \\ ${ }^{1}$ Department of Orthopaedic Surgery, Peking Union Medical College Hospital, Peking Union Medical College and Chinese Academy of Medical \\ Sciences, Beijing, China; ${ }^{2}$ Department of Pathology, Peking Union Medical College Hospital, Chinese Academy of Medical Science \& Peking Union \\ Medical College, Beijing, China \\ Contributions: (I) Conception and design: X Zhou, S Liu, Y Liu; (II) Administrative support: X Zhou, S Liu, Y Liu; (III) Provision of study materials \\ or patients: Y Liu; (IV) Collection and assembly of data: X Zhou, S Liu, S Yao, Z Huo; (V) Data analysis and interpretation: X Zhou, S Liu, S Yao, \\ Y Wang, Y Liu; (VI) Manuscript writing: All authors; (VII) Final approval of manuscript: All authors. \\ "These authors contributed equally to this work. \\ Correspondence to: Yong Liu. Department of Orthopaedic Surgery, Peking Union Medical College Hospital, Peking Union Medical College and \\ Chinese Academy of Medical Sciences, No. 1 Shuaifuyuan Wangfujing, Beijing 100730, China. Email: liuyong_pumch@163.com.
}

Background Limited case reports of metastatic spinal bladder cancer (MSBC) have been published to date. Owing to the rarity of this condition, it has not been well-studied and it is thus difficult to predict patient prognosis or to plan appropriate clinical treatment strategies for MSBC. This study is by far the largest clinical case series on MSBC worldwide.

Methods: Six patients with MSBC were included from January 2010 to May 2020 at the bone tumor center of orthopedics department in Peking Union Medical College Hospital. Clinical information, radiological data, operative notes, and pathological results of all patients were reviewed. Baseline clinical data of all patients were retrospectively analyzed, and regular follow-up was performed postoperatively. Overall survival (OS) was the time from the initial spinal surgery to the death of patients or the end of May 2020, whichever came first.

Results: All six patients with MSBC were male patients, with an average age of $68.1 \pm 12.8$ years. The mean interval between surgery for primary BC and the first discovery of spinal metastases was 15.6 [2-33] months. Overall, nine spinal operations were performed in the six patients. The mean follow-up period was $11.0 \pm 4.2$ (range, 7-18) months. All patients (100\%) died from MSBC during the follow-up period, with a mean OS of $11.0 \pm 4.2$ (range, 7-18) months.

Conclusions: Patients with MSBC had a poor prognosis in this study. Spinal surgery combined with adjuvant therapy may contribute to relieving the clinical symptoms and improve the quality of life of patients. Appropriate surgical treatment options should be selected according to patients' general condition and relevant characteristics of spinal metastases.

Keywords: Bladder cancer; clinical features; prognosis; retrospective study; spinal metastasis; surgical treatment

Submitted Oct 05, 2020. Accepted for publication Jan 19, 2021.

doi: 10.21037/apm-20-1980

View this article at: http://dx.doi.org/10.21037/apm-20-1980 


\section{Introduction}

Bladder cancer (BC) is recognized as one of the most common malignancies of the urogenital system $(1,2)$. Around the world, the incidence of $\mathrm{BC}$ ranked $12^{\text {th }}$ among all types of malignant tumors in 2018 and ranked $6^{\text {th }}$ among all malignancies in male patients (3). In China, the incidence of $\mathrm{BC}$ in male patients ranks seventh among systemic tumors, thus the incidence has gradually increased in recent years, and the diseased population is increasingly becoming younger (4).

Metastasis to important visceral organs, such as the lung and liver, is common among patients with BC; however, metastasis to the spinal cord is relatively rare, and the condition is often ignored by clinicians $(1,5)$, who tend to pay more attention to the progression of primary bladder tumors and ignore the occurrence and development of bone metastases. Bone metastases of bladder cancer are often occult but develop rapidly, leading to the occurrence of bone symptoms and bone-related events. Thus far, there have been only a few reports of sporadic cases of metastatic spinal bladder cancer (MSBC), and limited experience has been accumulated to determine appropriate treatment strategy. However, MSBC can greatly affect the survival status of patients and result in the occurrence of skeletalrelated events and even catastrophic consequences (6).

To further understand the clinical features, treatment, and prognosis of MSBC, in this study, we reviewed and summarized clinical data of six patients with MSBC who were admitted to our department during the past 10 years. This means that among more than 1,000 patients with spinal metastases who underwent surgical treatment at our department, spinal metastatic lesions originated from bladder tumors in only six patients. The analysis results from these patients are reported in this study.

We present the following article in accordance with the STROBE reporting checklist (available at http://dx.doi. org/10.21037/apm-20-1980).

\section{Methods}

Six consecutive patients with MSBC were enrolled in this study from January 2010 to May 2020 at the bone tumor center of our department. Clinical information, radiological data, operative notes, and pathological results of all patients were reviewed. The final follow-up visits were conducted until May 2020. The study was conducted in accordance with the Declaration of Helsinki (as revised in 2013). The study was approved by institutional ethics board of Peking Union Medical College Hospital (No. S-K1268) the registration number of ethics board) and individual consent for this retrospective analysis was waived.

All six patients underwent surgical treatment for primary $\mathrm{BC}$ before admission to our department, and the diagnosis of $\mathrm{BC}$ was confirmed by postoperative pathology. The clinical diagnosis of MSBC is made based on the following three criteria: (I) imaging findings (radiography, computed tomography (CT), magnetic resonance imaging (MRI), bone scan, positron emission tomography (PET)/CT are consistent with spinal metastasis, (II) a clear previous history of BC, and (III) diagnosis using pathological specimens of surgically removed spinal lesions. The American Spinal Injury Association (ASIA) impairment scale and Frankel score were used to assess the preoperative neurological status. The Eastern Cooperative Oncology Group (ECOG) score, visual analog scale (VAS) score, and Karnofsky score were applied to evaluate the quality of life of patients. Spinal stability was classified according to the Spinal Instability Neoplastic Score (SINS) system, and patients' prognosis and development of surgical procedures were initially assessed using the revised Tokuhashi and Tomita scoring systems. Individualized surgical treatment was completed by the surgical team led by Professor Yong Liu.

Postoperatively, radiological re-examination (spinal radiography, CT, or MRI) was regularly performed at 3 and 6 months and at regular intervals every 6 months during the subsequent follow-up period. For further evaluation of patients showing spinal tumor progression, PET/CT was highly recommended. Follow-up data were obtained via outpatient visits or telephonic interviews. The VAS score and Frankel and Karnofsky scoring systems were applied to reassess the postoperative neurological function and quality of life of patients during the followup period. The overall survival (OS) was defined as the time duration between the date of spinal operation and the date of final death or the last follow-up visit in January 2020, whichever came first.

\section{Statistical analysis}

The SPSS 23.0 software was used for analyzing statistical data. Continuous variables are expressed as frequencies and proportions. 


\section{Results}

\section{Clinical features}

Table 1 presents the clinical characteristics and surgical information of all six patients who underwent a total of nine operations. These patients with MSBC were all male patients, with an average age of $68.1 \pm 12.8$ (range, 46-83) years. All patients underwent surgical procedure for primary $\mathrm{BC}$, of whom five underwent total cystectomy for myometrial invasive $\mathrm{BC}$ (MIBC) and one underwent transurethral resection of non-muscular invasive $\mathrm{BC}$ (NMIBC). The mean interval between the initial diagnosis of $\mathrm{BC}$ and the final diagnosis of MSBC was 15.6 (range, 2-33) months. Three patients had multiple pelvic lymph node metastases, two had multiple extraspinal bone metastases, and no patients had clear lung, liver, and other organ metastases. After undergoing the initial surgery for BC, six patients agreed to undergoing postoperative chemotherapy. Among them, only one patient received chemoradiotherapy and another patient received chemotherapy combined with cellular immunotherapy.

All these patients exhibited gradually increased back pain before spinal surgery. On admission, one patient had incomplete paralysis of both the lower extremities, and the other five patients could walk by themselves. The mean duration of symptoms before spinal surgery was $2.6 \pm$ 2.3 (range, 0.25-7) months. In four patients, the lesions were located in the thoracic spine, and in five patients, these were located in the lumbar spine. The scoring information for spinal metastases in all patients are shown in Table 2. It was difficult to distinguish MSBC from spinal metastases of other malignancies on imaging because most of them present with osteolytic changes. Osteolytic spinal lesions could be observed in five patients, whereas the spinal metastases showed mixed changes in one patient. In four patients, spinal cord compression occurred, and two patients exhibited obvious paravertebral soft tissue masses. Three patients had single vertebral body metastasis (Figure 1) and three had multiple vertebral body metastases (Figure 2).

Surgical treatment was completed by the team of Professor Liu at our department, with a total of nine spinal operations. Four patients underwent posterior tumor resection, spinal cord or nerve root decompression, and internal fixation. During the operations, the mean blood loss was 1,125 (range, 500-1,500) mL. Percutaneous vertebroplasty using bone cement was performed in five patients, with an average intraoperative blood loss of 48 (range, 30-80) $\mathrm{mL}$. No definite complications were noted in any patient during the perioperative period. Postoperatively, all patients continued to receive the established adjuvant therapies. All six patients were recommended to undergo bisphosphonate therapy after spinal surgery. Our pathological findings supported the accurate final diagnosis of MSBC.

\section{Follow-up}

The mean follow-up period was $11.0 \pm 4.2$ (range, 7-18) months. All six patients (100\%) died from MSBC during the follow-up period, with a mean OS of $11.0 \pm 4.2$ (range, 7-18) months. Postoperatively, all patients' symptoms improved significantly. One week after surgery, assessment of the neurological status revealed grade 1 improvement based on the Frankel score in the patient with incomplete paralysis before spinal surgery. During the perioperative period, no obvious complications were noted.

\section{Discussion}

$\mathrm{BC}$ is reported to be the one of the most common urinary tract tumors globally $(1,2)$. The metastatic pathways mainly include lymphatic metastasis, hematological metastasis, and direct seeding metastasis. The treatment of metastatic BC has always been clinically difficult (2,7-9). The median survival time is approximately $12-14$ months, and the prognosis is far worse than that of kidney and prostate cancer (2,7-9). MSBC has a relatively rare incidence, and together with its poor prognosis, it is usually not paid sufficient attention by clinicians. The previous literature has only been reported in sporadic case reports or case series $(5,10)$. Under these circumstances, it is difficult for clinicians to recommend the appropriate treatment strategy and accurately evaluate the prognosis.

Some studies have shown that early BC is mainly caused by lymphatic metastasis $(7-9,11)$. Factors influencing the lymphatic metastasis of BC mainly include the ability of cancer cells to invade and migrate, structure of lymphatic vessels, and mechanism of important biological regulatory molecules on cancer cells $(7-9,11)$. NMIBC accounts for approximately $75 \%$ of BCs, of which $50 \%$ is of low grade, with the tumors majorly exhibiting a micropapillary pattern, and the 5 -year survival rate is $90 \%$, but with a high recurrence rate. MIBC accounts for approximately $25 \%$ of all BC cases, and most of these are of high-grade (12-18). The tumors mainly exhibit mixed shapes, and half of them develop into metastatic tumors. The 5 -year survival 


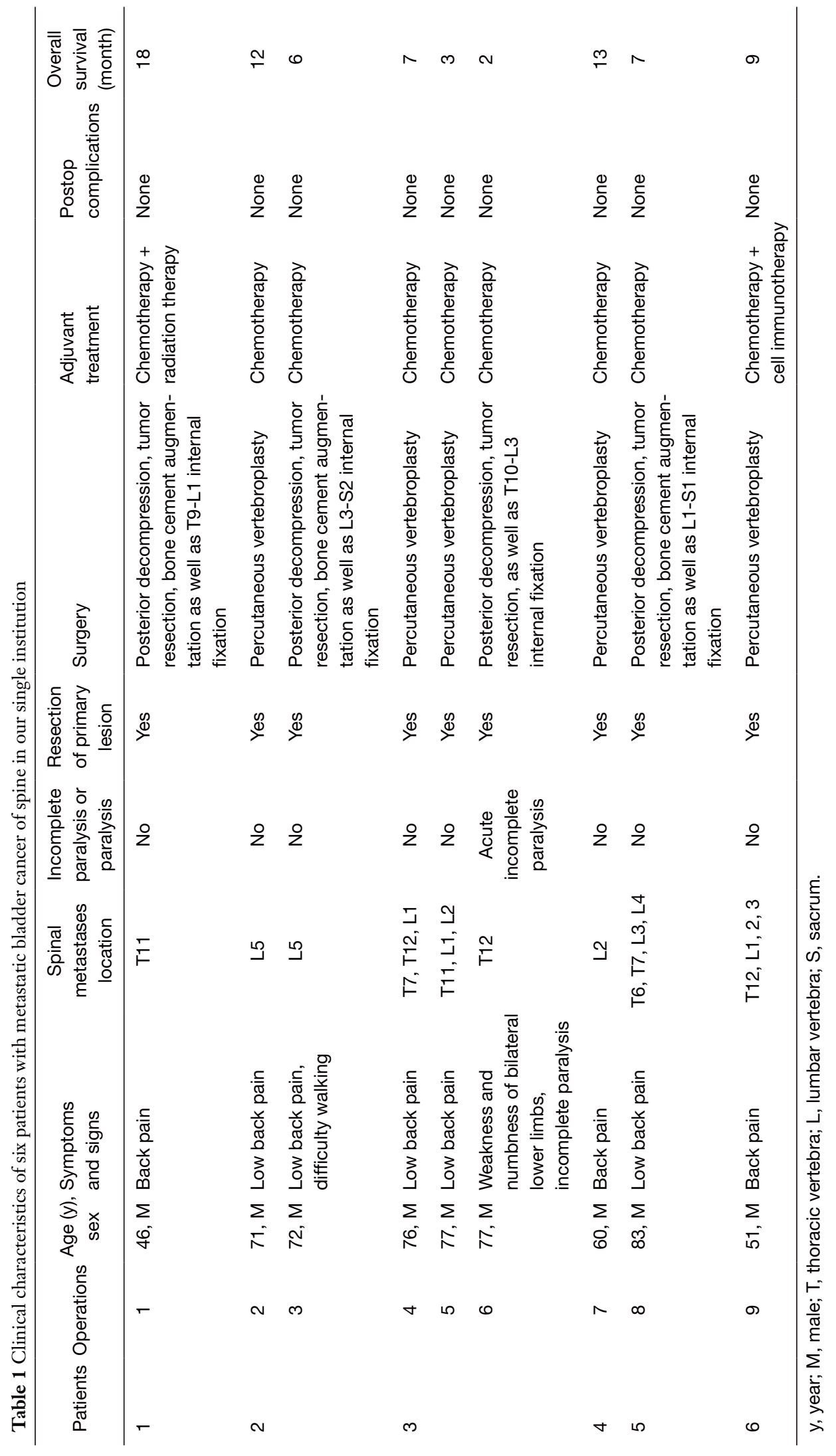




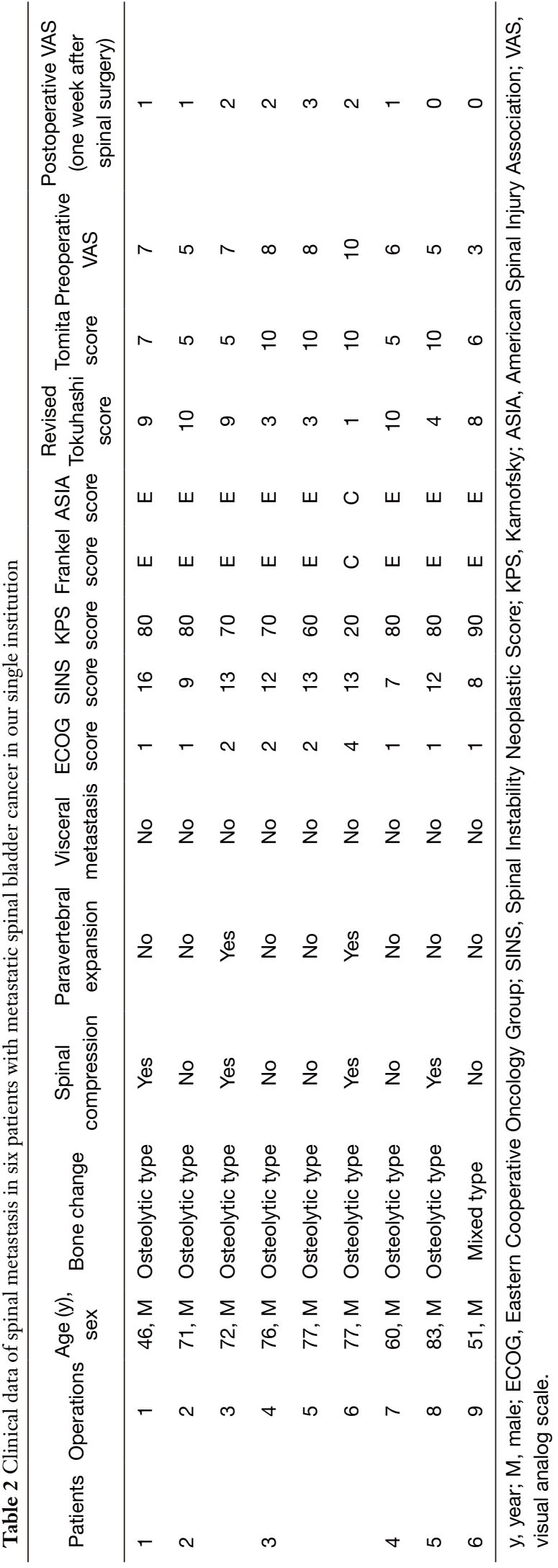

rate is reported to be only $5 \%$. BC is more common in men aged $>65$ years; however, in recent years the age of onset has tended to be younger, and the ratio of men to women ranges from $3: 1$ to $4: 1(1,2)$. BC has the characteristics of easy invasion, recurrence, drug resistance, and multifocality. During the disease course, $10-30 \%$ of cases of NMIBC can progress to MIBC (12-18). Currently, the main treatment method for NMIBC is simple transurethral resection of bladder tumor (TURBT) and TURBT combined with bladder perfusion therapy (19-21). Due to the diversity of associated patient factors, the specific treatment methods also vary.

Because MSBC is prone to missed diagnosis and misdiagnosis in clinical practice, its accurate diagnosis has become an important issue for clinicians. According to our experience of diagnosis and treatment at a single center, we believe that the following three points should be valued: (I) for patients with BC, close follow-up should be conducted after primary lesion resection, and bone scan should be regularly performed every 1-2 years postoperatively. (II) For patients with back pain, lower limb pain or numbness, decreased muscle strength of the lower limbs, obstructed defecation, and pathological fractures, the possibility of bone metastasis from $\mathrm{BC}$ should be considered during the diagnosis, and if necessary, MRI and nuclear medicine test should be conducted. (III) For patients with a confirm diagnosis of MSBC, it is necessary to perform lesion biopsy or surgical intervention as soon as possible, so that comprehensive treatment strategy can be formulated and the prognosis of patients can be improved as far as possible.

For patients with MSBC having incurable pain or neurologic impairment, spinal surgery is considered the most effective treatment option. Considering the functional status and prognosis of the patients, we applied four operations of open surgery and five operations of percutaneous vertebroplasty. During the operation, spinal metastases in MSBC was found to have richer blood flow than other spinal metastases, concurrent with the radiological characteristics. The spinal lesions were usually osteolytic and might be complicated by spinal cord compression, paravertebral soft tissue masses, and pathological vertebral compression fractures. Therefore, percutaneous vertebroplasty using bone cement might contribute to rebuilding spinal stability and improving patients' symptoms. The most common symptom caused by spinal metastasis is back pain or lower limb numbness; therefore, supportive treatment with analgesics is usually needed to improve pain. The results are often short-term 

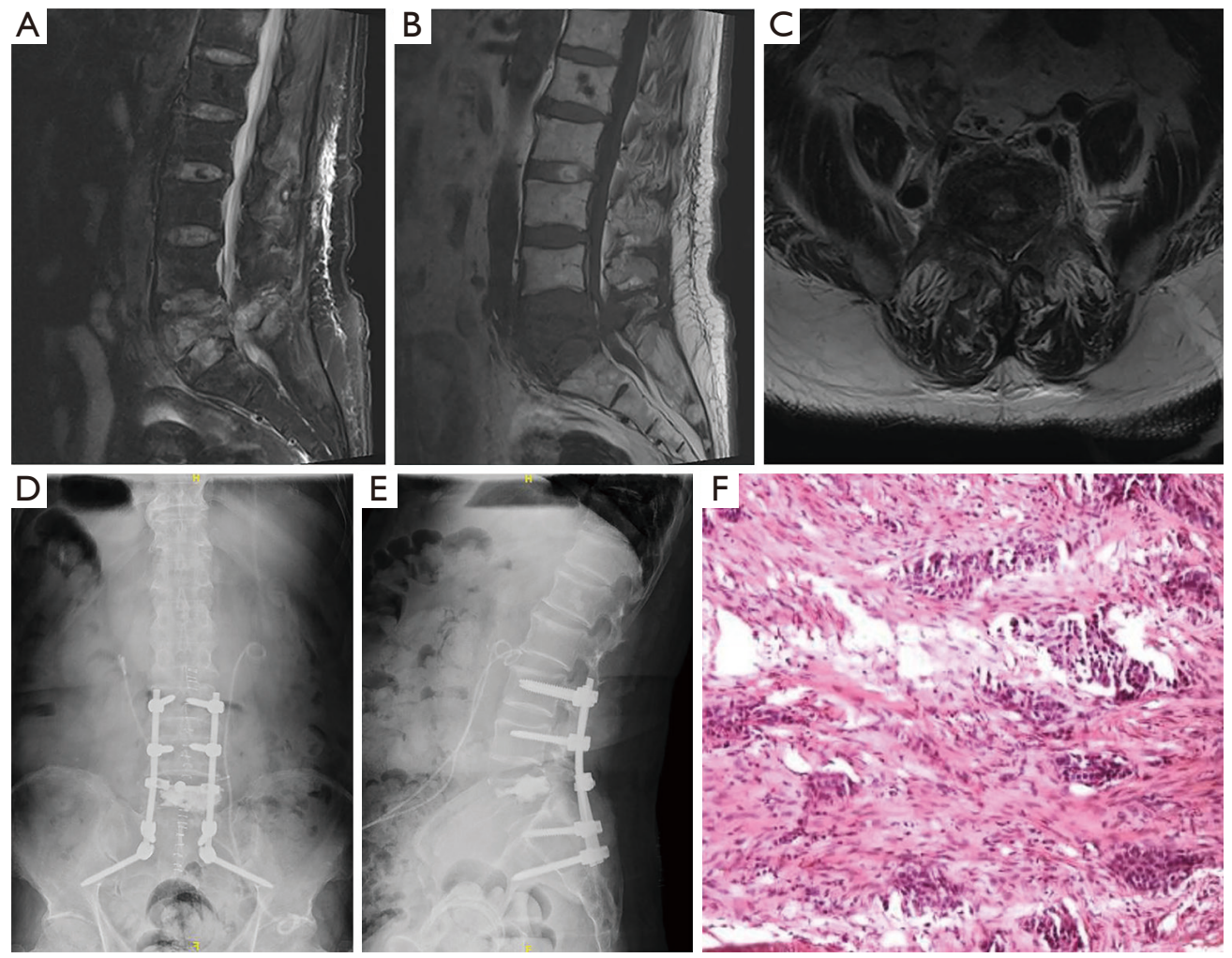

Figure 1 Radiographic and pathological images of a representative 72-year-old male patient (Case \#2). (A,B,C) Preoperative sagittal and transverse MRI revealing vertebral metastases of L5. (D,E) X-ray images of the lumbar spine obtained postoperatively. (F) Microphotography showing characteristic nests of tumor cells (H\&E, ×100). MRI, magnetic resonance imaging; L, lumbar vertebra.

and limited. Traditional open surgery to remove spinal metastases is more thorough and effective; however, the surgery itself can result in more complications. In addition, bone cement has been confirmed to have the potential of inhibiting tumors, the operation duration is short, patients recover quickly, and treatment compliance is good, which makes this technique promising and effective for the surgical treatment of MSBC.

Expected survival time is recognized as an important factor to determine whether to perform surgical treatment and to choose the appropriate procedure. Palliative treatment is more reasonable if the life expectancy is $<3$ months. The staging systems proposed by Tokuhashi et al. and Tomita et al. emphasized general information (primary tumor type) for prognosis evaluation to determine appropriate management strategy for patients with different scores $(22,23)$. However, the Tomita scoring system and revised Tokuhashi scoring system are not necessarily perfect for all spinal metastasis of a specific primary tumor, thereby affecting correct prognostic judgments and decisions of clinicians. In the scoring systems used in the present study, there were certain imperfections, as well as large space for refinement. For a specific type of primary tumor, the proposed spinal metastatic cancer scoring system would be much more valuable and accurate for clinical decisionmaking and improving patient prognosis.

In our study, adjuvant therapies for MSBC included chemotherapy, radiotherapy, cell immunotherapy, and bisphosphonate therapy (e.g., zoledronic acid). The major goal of treatment is to control local lesions, relieve symptoms, prevent pathological vertebral compression fractures, and ultimately improve quality of life of patients. In our study, all six patients underwent postoperative chemotherapy. Muscle invasive BC is characterized by high malignancy, complicated treatment, being prone to metastasis, and having poor prognosis. The 5 -year survival rate is usually $<50 \%$, which is a serious threat to patients' life $(1,2)$. Radical cystectomy with pelvic lymphadenectomy remains the gold standard for MIBC $(24,25)$. The gemcitabine combined with cisplatin (GC) regimen is currently the most widely used 

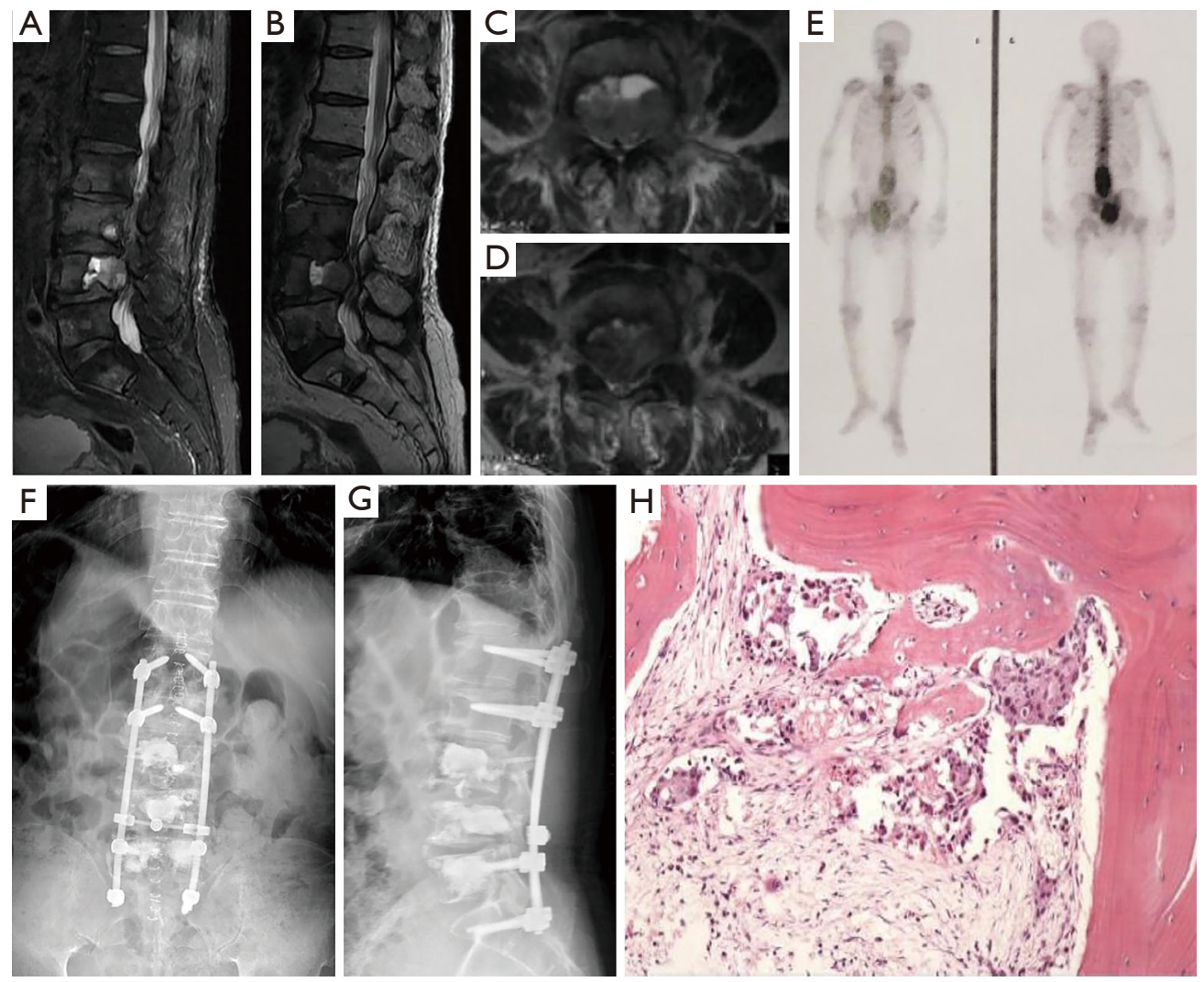

Figure 2 Radiographic and pathological images of a representative 83-year-old male patient (Case \#5). (A-D) Preoperative MRI scan revealing multiple vertebral metastases. (E) Bone scan revealing suspected spinal metastases. (F,G) Postoperative X-rays of the lumbar spine. (H) Microphotography showing significant nuclear pleomorphism with prominent nucleoli (H\&E, $\times 100)$. MRI, magnetic resonance imaging.

first-line chemotherapy regimen $(18,19)$. The treatment options and prognosis are very different for NMIBC and MIBC; NMIBC is characterized by a high recurrence rate and a low mortality rate, and the treatment is mainly assisted by intravesical instillation of the Bacillus Calmette-Guérin vaccine or chemotherapy after local resection, whereas MIBC mainly requires comprehensive surgery, chemotherapy, and radiotherapy, and approximately $50 \%$ of the patients have local recurrence within 2 years after radical surgery for BC depending on the pathological stage and lymph node status of the primary BC $(19-21,24,25)$. For MIBC, local recurrence accounts for $30 \%$ of all recurrences, and distant metastases are much more common. Approximately $10-15 \%$ of patients with MIBC already have metastases at the time of diagnosis of recurrence $(19-21,24,25)$. The efficacy of bisphosphonate therapy can reduce the risk of pathological fractures in the spine, long bones, pelvis, and extraspinal sites in patients with metastatic bone cancer (26). All six patients enrolled in this study were recommended to receive bisphosphonate therapy. Due to the limited number of cases and lack of clinical attention, the definite efficacy of bisphosphonate therapy in patients with MSBC is unclear (27).

Some limitations of the present study should be discussed. First, our study included very few patients for a detailed statistical analysis to evaluate the prognostic factors of MSBC. Second, this study was conducted as a retrospective analysis at a single center, and the clinical application value needs to be further explored in a larger group of patients. More clinical work on this disease condition is required in future in order to continue to improve and guide clinical diagnosis more accurately. In addition, only patients with MSBC who received spine surgery within the past 10 years were included and analyzed in this study, and patients who chose palliative treatment alone were not selected, which may have brought a certain selective bias within the reached conclusions. Although only six patients were analyzed, this 
case series constituted the first clinical series of MSBC reported separately, and this study was based on more than 1,000 patients who underwent spinal surgery during the past 10 years. The retrospective analysis of data focuses on the clinical features and treatment strategy of MSBC, which will help accumulate experience and evidence to improve the management of this entity and reduce the incidence of perioperative complications. Multicenter prospective study with a larger sample size should be conducted to maximize the quality of life and disease and improve the prognosis of these patients.

\section{Conclusions}

With the prolongation of survival time of patients with BC, the incidence of bone metastases has gradually increased in recent years. Although the prognosis of patients with $\mathrm{BC}$ is poor, inappropriate palliative treatment might further delay the operative timing for MSBC, resulting in irreversible and even life-threatening consequences. Therefore, sufficient attention should be paid to the timely diagnosis and proper treatment of MSBC by clinicians. Open surgery is recommended as the preferred treatment option for patients with unrelieved pain or numbness caused by neurological impairment. Patients with MSBC should be closely monitored after spinal surgery in order to identify the progression of spinal lesions and reduce patients' symptoms and the occurrence of serious complications such as pathological fractures.

\section{Acknowledgments}

We would like to thank our colleagues at the Departments of Urology surgery, Anesthesiology, Pathology, Nuclear Medicine, Radiology, and Orthopaedic Surgery for their support.

Funding: This study was supported by Peking Union Medical College Graduate Student Innovation Fund [2018] (Project No. 2018-1002-02-08; Grant recipient: S.L.). The funder had no role in study design, data collection and analysis, decision to publish, or preparation of the manuscript.

\section{Footnote}

Reporting Checklist: The authors have completed the STROBE reporting checklist. Available at http://dx.doi. org/10.21037/apm-20-1980
Data Sharing Statement: Available at http://dx.doi. org/10.21037/apm-20-1980

Conflicts of Interest: All authors have completed the ICMJE uniform disclosure form (available at http://dx.doi. org/10.21037/apm-20-1980). The authors have no conflicts of interest to declare.

Etbical Statement: The authors are accountable for all aspects of the work in ensuring that questions related to the accuracy or integrity of any part of the work are appropriately investigated and resolved. The study was conducted in accordance with the Declaration of Helsinki (as revised in 2013). The study was approved by institutional ethics board of Peking Union Medical College Hospital (No. S-K1268) the registration number of ethics board) and individual consent for this retrospective analysis was waived.

Open Access Statement: This is an Open Access article distributed in accordance with the Creative Commons Attribution-NonCommercial-NoDerivs 4.0 International License (CC BY-NC-ND 4.0), which permits the noncommercial replication and distribution of the article with the strict proviso that no changes or edits are made and the original work is properly cited (including links to both the formal publication through the relevant DOI and the license). See: https://creativecommons.org/licenses/by-nc-nd/4.0/.

\section{References}

1. Flaig TW, Spiess PE, Agarwal N, et al. Bladder Cancer, Version 3.2020, NCCN Clinical Practice Guidelines in Oncology. J Natl Compr Canc Netw 2020;18:329-54.

2. Alfred Witjes J, Lebret T, Comperat EM, et al. Updated 2016 EAU Guidelines on Muscle-invasive and Metastatic Bladder Cancer. Eur Urol 2017;71:462-75.

3. Bray F, Ferlay J, Soerjomataram I, et al. Global cancer statistics 2018: GLOBOCAN estimates of incidence and mortality worldwide for 36 cancers in 185 countries. CA Cancer J Clin 2018;68:394-424.

4. Chen W, Sun K, Zheng R, et al. Cancer incidence and mortality in China, 2014. Chin J Cancer Res 2018;30:1-12.

5. Crowley RW, Sherman JH, Le BH, et al. Intramedullary spinal cord metastasis from bladder carcinoma: case report. Neurosurgery 2008;63:E611-2; discussion E612.

6. Tsuda Y, Nakagawa T, Shinoda Y, et al. Skeletal-related events and prognosis in urothelial cancer patients with 
bone metastasis. Int J Clin Oncol 2017;22:548-53.

7. Flaig TW. NCCN Guidelines Updates: Management of Muscle-Invasive Bladder Cancer. J Natl Compr Canc Netw 2019;17:591-3.

8. Thoma C. Bladder cancer: Better prediction of lymph node metastasis. Nat Rev Urol 2016;13:301.

9. Seiler R, Lam LL, Erho N, et al. Prediction of Lymph Node Metastasis in Patients with Bladder Cancer Using Whole Transcriptome Gene Expression Signatures. J Urol 2016;196:1036-41.

10. Abdulazim A, Backhaus M, Stienen MN, et al. Intramedullary spinal cord metastasis and multiple brain metastases from urothelial carcinoma. J Clin Neurosci 2011;18:1405-7.

11. Sharma M, Goto T, Yang Z, et al. The impact of perivesical lymph node metastasis on clinical outcomes of bladder cancer patients undergoing radical cystectomy. BMC Urol 2019;19:77.

12. Cheng L, Davison DD, Adams J, et al. Biomarkers in bladder cancer: translational and clinical implications. Crit Rev Oncol Hematol 2014;89:73-111.

13. Elsen S, Lerut E, Van Cleynenbreugel B, et al. Biodistribution of Evans blue in an orthotopic AY-27 rat bladder urothelial cell carcinoma model: implication for the improved diagnosis of non-muscle-invasive bladder cancer (NMIBC) using dye-guided white-light cystoscopy. BJU Int 2015;116:468-77.

14. Christensen E, Birkenkamp-Demtroder K, Nordentoft I, et al. Liquid Biopsy Analysis of FGFR3 and PIK3CA Hotspot Mutations for Disease Surveillance in Bladder Cancer. Eur Urol 2017;71:961-9.

15. Flaig TW, Spiess PE, Agarwal N, et al. NCCN Guidelines Insights: Bladder Cancer, Version 5.2018. J Natl Compr Canc Netw 2018;16:1041-53.

16. Lei T, Zhao X, Jin S, et al. Discovery of potential bladder cancer biomarkers by comparative urine proteomics and analysis. Clin Genitourin Cancer 2013;11:56-62.

17. Spiess PE, Agarwal N, Bangs R, et al. Bladder Cancer,

Cite this article as: Zhou X, Liu S, Yao S, Huo Z, Wang Y, Liu Y. Clinical features and management experience in patients with metastatic spinal bladder cancer: a single-institution 10year retrospective study. Ann Palliat Med 2021;10(4):3817-3825. doi: 10.21037/apm-20-1980
Version 5.2017, NCCN Clinical Practice Guidelines in Oncology. J Natl Compr Canc Netw 2017;15:1240-67.

18. Robinson AG, Wei X, Mackillop WJ, et al. Use of Palliative Chemotherapy for Advanced Bladder Cancer: Patterns of Care in Routine Clinical Practice. J Natl Compr Canc Netw 2016;14:291-8.

19. Winquist E, Kirchner TS, Segal R, et al. Neoadjuvant chemotherapy for transitional cell carcinoma of the bladder: a systematic review and meta-analysis. J Urol 2004;171:561-9.

20. Herr HW. Role of Repeat Resection in Non-MuscleInvasive Bladder Cancer. J Natl Compr Canc Netw 2015;13:1041-6.

21. Abern MR, Owusu RA, Anderson MR, et al. Perioperative intravesical chemotherapy in non-muscle-invasive bladder cancer: a systematic review and meta-analysis. J Natl Compr Canc Netw 2013;11:477-84.

22. Tokuhashi Y, Matsuzaki H, Oda H, et al. A revised scoring system for preoperative evaluation of metastatic spine tumor prognosis. Spine (Phila Pa 1976) 2005;30:2186-91.

23. Tomita K, Kawahara N, Kobayashi T, et al. Surgical strategy for spinal metastases. Spine (Phila Pa 1976) 2001;26:298-306.

24. Witjes JA, Bruins HM, Cathomas R, et al. European Association of Urology Guidelines on Muscle-invasive and Metastatic Bladder Cancer: Summary of the 2020 Guidelines. Eur Urol 2021;79:82-104.

25. Kessler ER, Kukreja JB, Geiger CL, et al. Treating Elderly Patients With Muscle-Invasive Bladder Cancer. J Natl Compr Canc Netw 2020;18:783-90.

26. Alcaraz A, Gonzalez-Lopez R, Morote J, et al. Biochemical markers of bone turnover and clinical outcome in patients with renal cell and bladder carcinoma with bone metastases following treatment with zoledronic acid: The TUGAMO study. Br J Cancer 2013;109:121-30.

27. Climent MA, Anido U, Mendez-Vidal MJ, et al. Zoledronic acid in genitourinary cancer. Clin Transl Oncol 2013;15:871-8. 